\section{$\mathrm{Al}(\mathrm{OH})_{3}$ - die Mischung macht's}

S eit Oktober 2009 steht mit Avanz ${ }^{\circledR}$ ein neues immunologisch verstärktes Präparat zur subkutanen spezifischen Immuntherapie zur Verfügung. Durch ein optimiertes Verhältnis von Allergen zum Depotträger $\mathrm{Al}(\mathrm{OH})_{3}$ können die Aufdosierungsphase verkürzt und die Erhaltungsdosis reduziert werden. Aluminiumhydroxid wird bei der Hyposensibilisierung wegen seines Depoteffekts schon lange eingesetzt. Eine aktuelle Untersuchung hat nun gezeigt, dass auch die Immunogenität eines Allergenpräparates entscheidend vom Aluminiumhydroxid-Allergen-Verhältnis abhängt: Wird nur Allergen allein verabreicht, erfolgt eine vergleichsweise geringe Produktion von spezifischen Antikörpern. Steigert man die $\mathrm{Al}(\mathrm{OH})_{3}-$ Konzentration bis zu einem bestimmten Grad, erhöht sich auch die Antikörperproduktion.

Beim neuen Präparat Avanz ${ }^{\circledR}$ wurde das Verhältnis von Allergen zu Aluminiumhydroxid auf etwa 1:3 optimiert. Im Vergleich zu Präparaten, bei denen das Verhältnis Allergen zu Aluminiumhydroxid 1:1 beträgt, konnte das erforderliche Injektionsvolumen von $1 \mathrm{ml}$ auf $0,5 \mathrm{ml}$ reduziert werden, so dass insgesamt sowohl Allergendosis als auch absolut injizierte Menge an Aluminiumhydroxid reduziert werden konnten. Für den Patienten bringt das wesentliche Vorteile: Neben der guten Verträglichkeit ist die Therapie aufgrund des reduzierten Injektionsvolumens angenehmer. Zudem ermöglicht Avanz ${ }^{\circledR}$ eine schnellere Aufdosierung. Sie erfolgt während der ersten vier Wochen je einmal wöchentlich, in der fünften Woche ist bereits die Erhaltungsdosis erreicht. Drei Jahre lang wird dann diese Dosis in Intervallen von vier bis acht Wochen injiziert. Avanz ${ }^{\circledR}$ ist für die am häufigsten benötigten Allergene Birke, Frühblühermischung, Gräsermischung und Roggen, Wiesenlieschgras und Hausstaubmilben verfügbar.

Nach Informationen von ALK-Abelló, Wedel

\title{
Tablette mit 15 Jahren Praxiserfahrung
}

B islang standen in Deutschland zur Hyposensibilisierung bei Allergien aufgrund einer Sensibilisierung gegen Hausstaubmilben nur Injektionspräparate oder Tropfen zur Verfügung. Mit den LAIS $^{\circledR}$-Sublingualtabletten gibt es jetzt erstmals auch eine spezifische Immuntherapie in Tablettenform.

Wirksamkeit und Verträglichkeit des Präparats wurden in mehreren klinischen Doppelblindstudien nachgewiesen. Daten zu diesen Sublingualtabletten wurden sogar in einem Lancet-Beitrag veröffentlicht. Es konnte eine Verbesserung der Symptomatik um bis zu 48,4\% gegenüber Plazebo belegt werden. Ebenso liegen Hinweise zur Dosisabhängigkeit dieser Immuntherapie, zur Langzeitwirkung und zur Verbesserung der Lebensqualität vor. Firmeninterne Daten zur Verträglichkeit der Tabletten beschreiben pro 2.000 Anwendungen lediglich eine leichte lokale Nebenwirkung.

Der Grund für diese deutlich geringeren Nebenwirkungsraten im Vergleich zu anderen Sublingualtabletten ist das patentierte Herstellungsverfahren: LAIS ${ }^{\circledR}$ Sublingualtabletten enthalten keine nativen Allergene, sondern chemisch modifizierte, sogenannte carbamylierte Proteine (Monoide). Im Vergleich mit unmodifizierten Allergenen ergibt sich eine höhere Magensaftresistenz. Im Vergleich zu anderen Allergoiden bleibt darüber hinaus die molekulare Dimension des Monoids zusätzlich erhalten.

Vor über 15 Jahren in Italien von Lofarma entwickelt, steht mit LAIS $^{\circledR}$ ein klinisch validiertes und in der Praxis erprobtes Präparat zur Verfügung, mit dem mittlerweile über 300.000 Patienten außerhalb Deutschlands behandelt wurden.

Nach Informationen von Lofarma Deutschland, Willich

\section{Neuer Chef in Wedel}

Andreas H. Sander (52) ist seit dem 1. Oktober 2009 neuer Geschäftsführer der ALK-Abelló Arzneimittel $\mathrm{GmbH}$ in Wedel. Er ist außerdem verantwortlich für die ALK-Gesellschaften in Österreich und der Schweiz. Mit Sander gewinnt ALK einen Marketing-Experten mit großer Erfahrung in der Pharmaindustrie. Er studierte Pharmazie in Heidelberg sowie Berlin und Betriebswirtschaftslehre in den USA und war danach für verschiedene internationale Pharmaunternehmen tätig.

ALK ist weltweit Marktführer für Allergenpräparate zur Diagnostik und Therapie von Allergien. Das Unternehmen erhielt in den letzten Jahren mehrere Preise für innovative Produkte und Auszeichnungen als einer der besten Arbeitgeber Deutschlands sowie als besonders kundenorientierter Dienstleister.

Nach Informationen von ALK-Abelló, Wedel

\section{Kompass erleichtert Pråparatewahl}

Durch die TherapieallergeneVerordnung wurde die Zulassungspflicht für häufige Therapieallergene neu geregelt. Damit verbunden ist ein Wechsel weg von der Mischung kreuzreaktiver Allergene hin zur Monotherapie bzw. Behandlung mit Leitallergenen. Stallergenes bedient den Markt bereits zu $75 \%$ mit Präparaten, die der Therapieallergene-Verordnung entsprechen.

Um die Produktauswahl zu erleichtern, bietet das Unternehmen ab sofort den Therapie-Kompass Allergie an. Dieser bietet eine Navigationshilfe für die zukunftssichere Allergentherapie: Einfach am äußeren Ring die Diagnose einstellen schon erscheint die Therapieempfehlung, die den Vorgaben der neuen Verordnung entspricht. Ein Beispiel: Bei der Diagnose „Allergie gegen Birken-, Erlen-, Hasel- und Weißbuchenpollen" lautet die Therapieempfehlung Staloral ${ }^{\circledR} 300$ Birke. Denn die Birke ist das Leitallergen für die meisten frühblühenden Bäume.

Allergologen können den Therapie-Kompass Allergie kostenlos entweder über den Stallergenes-Außendienst oder direkt bei der Stallergenes $\mathrm{GmbH}$ unter Telefon (o 28 42) 90 40-1 46 bestellen.

Nach Informationen von Stallergenes, Kamp-Lintfort 